\title{
Le squelette de l'homme fossile d'Asselar (Mali, Holocène ancien) : observations archéothanatologiques
}

\author{
Archeothanatological Observations on the Human Skeleton from Asselar (Early Holocene, Mali)
}

\author{
J. Dumesnil · F. Valentin - A. Vialet \\ C Société d'Anthropologie de Paris et Lavoisier SAS 2017
}

\begin{abstract}
Résumé L'homme fossile d'Asselar, découvert en 1927 dans l'actuel Mali, n'a été que peu exploité depuis l'étude de Boule et Vallois (1932). Daté de l'Holocène ancien, il est, avec la série d'Hassi-el-Abiod, un témoin des populations vivant dans un Sahara alors « vert », bien différent de celui que nous connaissons aujourd'hui. L'hypothèse d'un dépôt funéraire, écartée dès la première étude au profit de celle d'une noyade et d'un enfouissement accidentel, est considérée pour la première fois selon les principes de l'anthropologie de terrain et grâce à l'imagerie médicale qui permet un accès complet et non invasif aux parties du squelette toujours incluses dans le sédiment.
\end{abstract}

Mots clés Mali · Sahara · Holocène ancien ·

Archéothanatologie $\cdot$ Imagerie 3D

\begin{abstract}
The fossilized human skeleton of Asselar was discovered in 1927 in present-day Mali. This specimen has been very little studied since it was first described by Boule and Vallois (1932). Dated to the early Holocene, it provides, together with the Hassi-el-Abiod series, evidence of the human groups who lived in then the fertile Sahara, which was very different from the desert we know today. The hypothesis of a deliberate burial, which was ruled out by the first study that considered it to be a case of drowning, is assessed for the first time in light of field anthropology principles and thanks to the medical imaging techniques which allowed full
\end{abstract}

\section{J. Dumesnil}

MNHN, UMR 7194, UPVD, 1 ,

rue René-Panhard, F-75013 Paris, France

F. Valentin

CNRS, UMR 7041, 21, allée de l'Université,

F-92023 Nanterre cedex, France

\section{A. Vialet $(\square)$}

MNHN, UMR 7194, UPVD, CERP, 1,

rue René-Panhard, F-75013 Paris, France

e-mail : amelie.vialet@mnhn.fr and non-invasive access to parts of the skeleton that are still naturally embedded in the sediment.

Keyword Mali $\cdot$ Sahara $\cdot$ Ancient Holocene · Archaeothanatology $\cdot 3 \mathrm{D}$ Imaging techniques

\section{Introduction}

Le squelette de l'homme d'Asselar a été découvert en 1927 lors de la mission Augiéras-Draper, dont le but était « d'aller vers l'inconnu » en traversant le Sahara entre Alger et Dakar [1]. En suivant les indications fournies par les militaires au nord du Mali, non loin de la frontière algérienne, deux membres de l'équipe (T. Monod et V. Besnard) ont en effet repéré, à proximité du poste d'Asselar, des ossements humains affleurants, qu'ils ont prélevés. Le contexte géologique et la faune associée permettent aujourd'hui d'attribuer le squelette d'Asselar à l'Holocène ancien (entre 9500 et 7000 BP) $[2,3]$. Il s'agit d'une époque à laquelle le Sahara était humide, présentant une faune et une flore différentes de l'actuel, comme en témoignent les cuvettes environnantes dont le fond contient une couche de marne grise à mollusques d'eau douce [4], indiquant la présence probable d'un lac.

Le squelette a été ramené à Paris et donné à l'Institut de paléontologie humaine où il a été confié pour étude à Marcellin Boule (1861-1942) et Henri-Victor Vallois (18891981). Il est constitué de "plusieurs blocs et de quelques os et fragments recueillis isolément à la surface du sol » [4]. Conformément au paradigme du moment, son étude a suivi une approche raciologique. Il a alors été mis en relation avec les Hottentots, les Bochimans d'Afrique du Sud et les hommes de Grimaldi, considérés comme des ancêtres de la « race noire » [5]. C'est sans doute par son inscription au service de tels paradigmes tombés en désuétude dans les années 1960 [6] que ce squelette a été oublié jusqu'à une période récente. En 2013, Vialet et al. [3] ont mené une étude historiographique et un réexamen général de ce spécimen, mettant en évidence l'absence de questionnement sur son 
éventuel contexte funéraire. En effet, la question d'un dépôt funéraire a été écartée par les découvreurs qui affirment dans leur « livre de bord » qu' «il n'y a aucun indice de sépulture » [1]. Par la suite, faisant référence au contexte géologique (paléolac), c'est une noyade qui est évoquée par Boule et Vallois pour expliquer la bonne conservation du squelette [4].

L'« anthropologie de terrain » permet de reconsidérer ces propositions et de préciser les conditions d'enfouissement de l'homme d'Asselar grâce à l'étude des relations spatiales entre les os formant une articulation [7-9]. Déjà, les observations réalisées par Vialet et al. en 2013 [3] avaient permis d'argumenter un dépôt primaire et un enfouissement rapide sur la base de la bonne conservation du squelette, de la position du corps et de la préservation des volumes principaux (thorax et bassin). Nous proposons ici de nous concentrer sur l'analyse des relations articulaires entre les os des pieds et de la main droite encore engagés dans un bloc de sédiment induré et donc maintenus dans la position de leur découverte. Pour cette analyse, nous avons eu recours au scanner médical et à l'imagerie 3D.

\section{Matériel}

Rappelons que l'ensemble du squelette est bien conservé même si les premiers auteurs précisent que «les eaux avaient emporté quelques débris et les avaient dispersés sur plusieurs mètres de la pente » [4]. Il est aujourd'hui constitué d'os isolés et d'os encore inclus dans la gangue gréseuse. Un bloc vertébral contient cinq cervicales ( $\mathrm{C} 2$ à $\mathrm{C} 6$ ), un autre comprend $\mathrm{C} 7$ et six vertèbres thoraciques et les portions postérieures des côtes droites, un troisième renferme les vertèbres thoraciques basses et les premières lombaires (T7 à L2), un quatrième contient les autres lombaires, le sacrum et l'ilium gauche, et un cinquième inclut les côtes gauches.

Au niveau des extrémités, deux blocs comprennent, l'un, le cinquième métacarpien droit ainsi qu'une partie du cunéiforme latéral droit et deux fragments des métatarsiens, et l'autre, la partie distale des tibias, l'extrémité distale de la fibula gauche, des os des deux pieds et de la main droite (Fig. 1). Un fragment de la fibula gauche (quart distal de la diaphyse) est détaché de ce dernier bloc qui est également cassé au niveau de la partie distale des pieds manquante. Ce bloc présente un intérêt considérable, car les os se trouvent encore dans la position qui était la leur lors de la découverte. Son examen donne la possibilité d'obtenir des indications sur l'état des connexions articulaires des extrémités du squelette d'Asselar.

\section{Méthode}

Afin de discuter des modalités de dépôt du squelette d'Asselar, nous nous sommes concentrés sur le bloc comprenant l'extrémité distale de la fibula gauche, des tibias et les os des pieds et de la main droite, pour lequel Boule et Vallois avaient effectué un inventaire [4] que nous avons complété

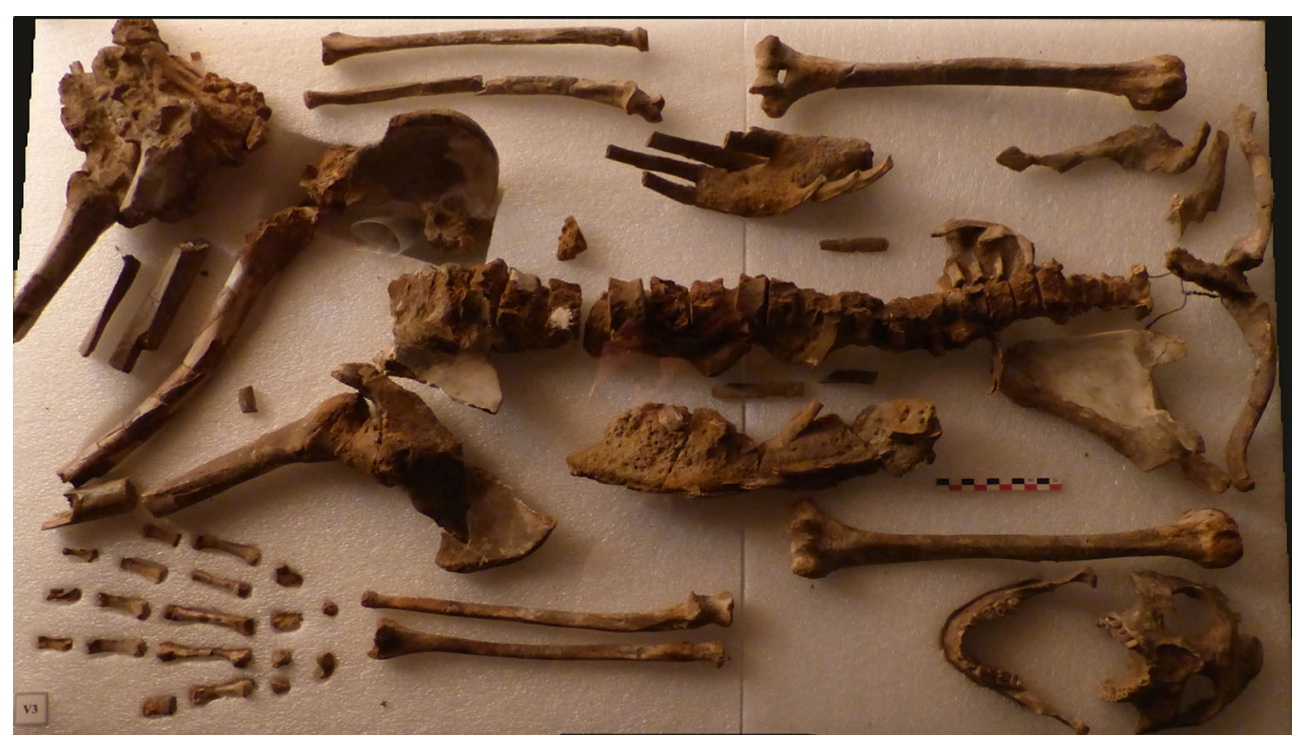

Fig. 1 Le squelette de l'homme d'Asselar tel qu'il est conservé à l'IPH, c'est-à-dire respectant la disposition donnée par Boule et Vallois pour les besoins photographiques de leur publication [4], en haut à gauche : le bloc comprenant les extrémités distales de la fibula gauche, des tibias, les os des pieds et de la main droite, qui a été tomographié (photographie A. Vialet) / The Asselar skeleton as preserved at the IPH in the position recommended by Boule and Vallois for photographic purposes [4]. Upper left: the block containing the distal ends of the left fibula, tibias and bones from the feet and the right hand. This block was CT-scanned (picture A. Vialet) 
grâce aux données tomographiques. Les paramètres techniques de ces dernières sont les suivants : épaisseur des coupes : $0,8 \mathrm{~mm}$; espace entre les coupes : $0,4 \mathrm{~mm} ; \mathrm{kVp}: 120$; X-Ray Tube Current : $108 \mathrm{~mA}$; taille des pixels : 0,37 $\times$ $0,37 \mathrm{~mm}$; largeur et hauteur : $285,00 \mathrm{~mm}$ (768 pixels). Une segmentation manuelle a été effectuée pour l'ensemble des 768 coupes en utilisant le logiciel Aviso 9.0.1, car les valeurs de densité entre l'os et la matrice se sont avérées trop proches pour appliquer une segmentation semi-automatique.

\section{Résultats}

\section{État de conservation}

Notons préalablement que nos déterminations anatomiques concordent avec celles des premiers auteurs [3]. De plus, les observations effectuées grâce à la tomographie sur le bloc contenant la fibula gauche, les tibias, les os des deux pieds et de la main droite permettent d'ajouter une phalange distale de la main droite au premier inventaire.

D'une façon générale, toutes les régions du squelette ainsi que les différents éléments osseux sont bien représentés. Les extrémités en particulier sont relativement complètes. Ainsi, pour la main gauche, seuls manquent deux os du carpe, deux phalanges moyennes et les cinq phalanges distales tandis que pour la main droite, quatre os du carpe ainsi que le deuxième métacarpien, et la majorité des phalanges n'ont pas été identifiés. Tous les tarses sont présents ainsi que, de façon fragmentaire, deux métatarses à droite et cinq à gauche. Les phalanges moyennes et distales ne sont pas conservées.

Ces absences relèvent sans doute principalement de dégradations taphonomiques remarquées dès la découverte et dues à l'affleurement du squelette à la surface du sol et au ruissellement des eaux de pluie [3].

\section{Bilan articulaire}

L'analyse du bloc grâce aux données tomographiques permet d'observer la bonne cohérence anatomique des éléments osseux composant les deux pieds et la main droite. Ainsi, l'articulation persistante de la cheville droite est maintenue (Fig. 2) ; la cheville gauche est en connexion lâche (Fig. 3). Les articulations talo-calcanéennes sont disjointes, alors que celles joignant les autres tarsiens sont maintenues. Les os du carpe sont en connexion stricte, et les fragments de métacarpiens, parallèles les uns aux autres (Fig. 4). Deux phalanges (moyenne et distale) de la main se succèdent selon la logique anatomique (Fig. 4).

Cette analyse permet également de définir les positions relatives des pieds et de la main droite. Ainsi, les chevilles sont étendues, et les deux pieds sont parallèles et rapprochés

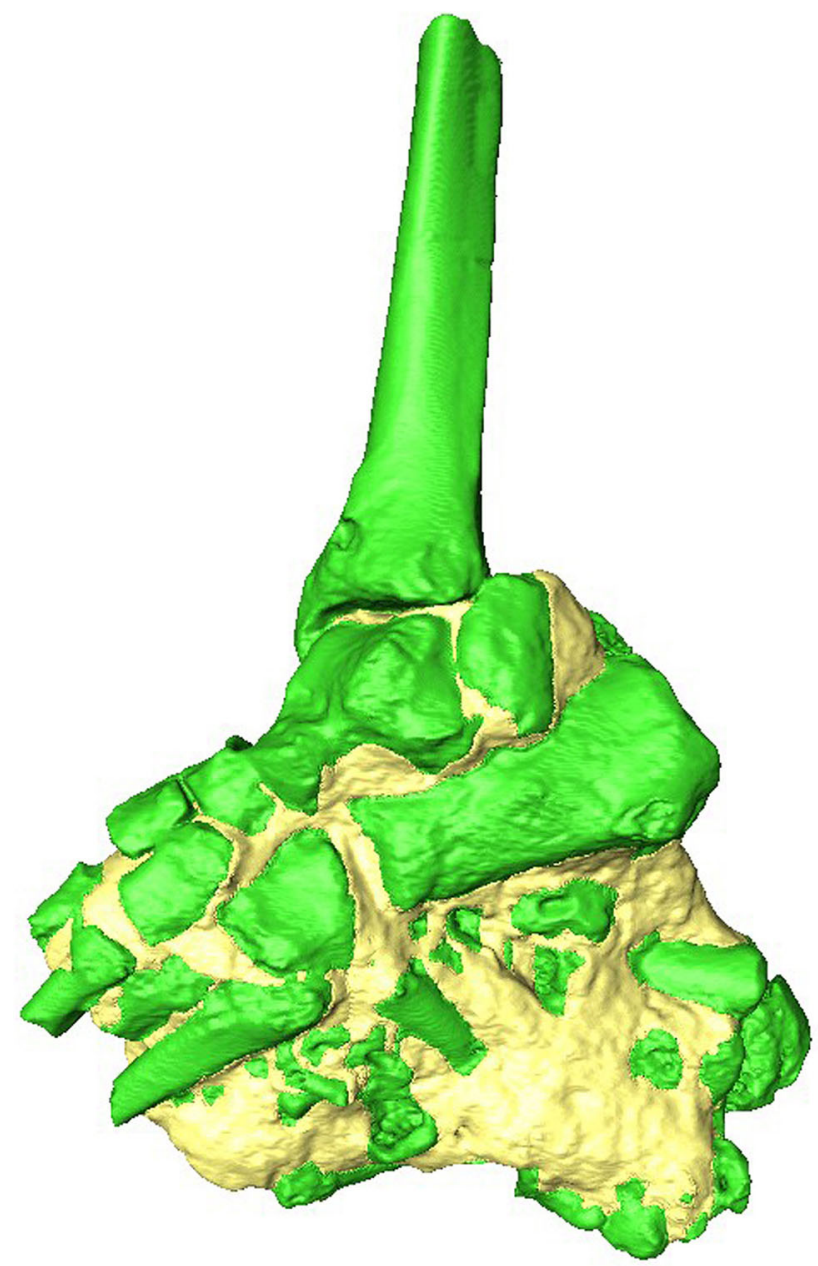

Fig. 2 Vue latérale gauche du bloc comprenant les extrémités distales de la fibula gauche, du tibia, les os des pieds et de la main droite après séparation par imagerie 3D entre les os (en vert) et la matrice gréseuse (en jaune). On distingue nettement dans la partie supérieure les tarses et la moitié proximale des métatarses du pied gauche, l'extrémité distale du tibia et la malléole de la fibula gauche. Capture d'écran sur Aviso 9.0.1 / Left lateral view of the block containing the distal ends of the left fibula and tibia and bones from the feet and the right hand, after virtual separation, by $3 \mathrm{D}$ imaging techniques, of the bones (green) from the matrix (yellow). The left foot can be clearly distinguished in the upper part: the tarsus and the proximal half of the metatarsus and the distal ends of the tibia and the fibula. Generated by Aviso 9.0.1

l'un de l'autre, le pied droit en position basse par rapport au gauche. La main droite, phalanges fléchies, est au contact du pied droit, sa face dorso-latérale se situant contre la face latéroplantaire du pied (Figs 3, 4). Cet arrangement est compatible avec la flexion des hanches visible sur la figure 1 et encore plus nette dans la monographie de Boule et Vallois [4]. L'association main droite-pieds implique de plus une hyperflexion latérale des genoux ramenant les pieds vers le bassin. 


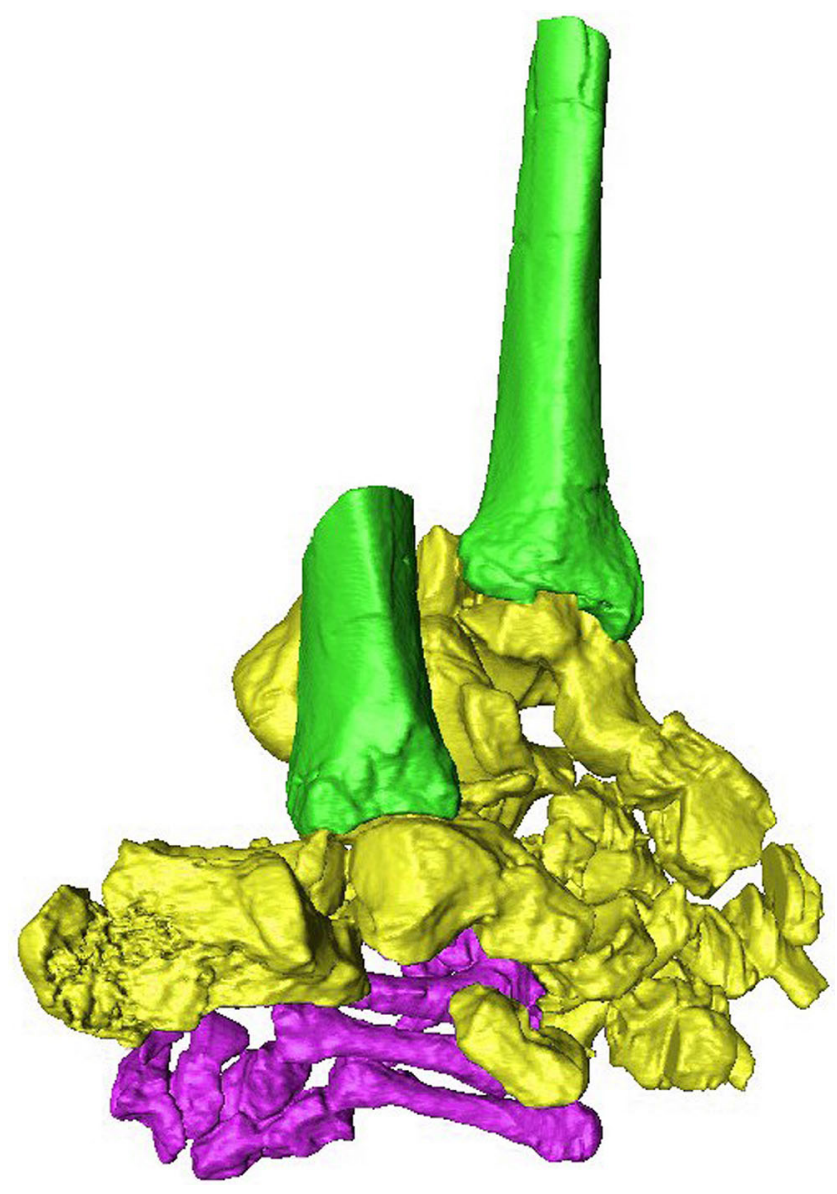

Fig. 3 Vue latérale droite du bloc comprenant les extrémités distales, des tibias, les os des pieds et de la main droite après dégagement virtuel des ossements par imagerie 3D. Tibias (en vert), pieds (en jaune), main droite (en violet). Capture d'écran sur Aviso 9.0.1 / Right lateral view of the block containing the distal ends of the tibias and bones from the feet and the right hand, virtually removed from the matrix by $3 \mathrm{D}$ imaging techniques. Tibias (green), foot (yellow), right hand (purple). Generated by Aviso 9.0.1

\section{Discussion}

Depuis la reprise de l'étude du squelette d'Asselar par Vialet et al. en 2013 [3], la question posée est celle des conditions de dépôt et d'enfouissement de cet individu. Boule et Vallois [4], dont la publication ne traite que très peu de cette interrogation, avaient émis l'hypothèse d'une noyade pour expliquer la bonne conservation du squelette. Or, cette proposition recouvre deux notions qui ne sont pas discutées à l'époque : celle des conditions d'enfouissement et celle de la cause de la mort. Les caractéristiques observées pour cet individu (bonne représentation du squelette, maintien en connexion d'articulations labiles et conservation des volu-

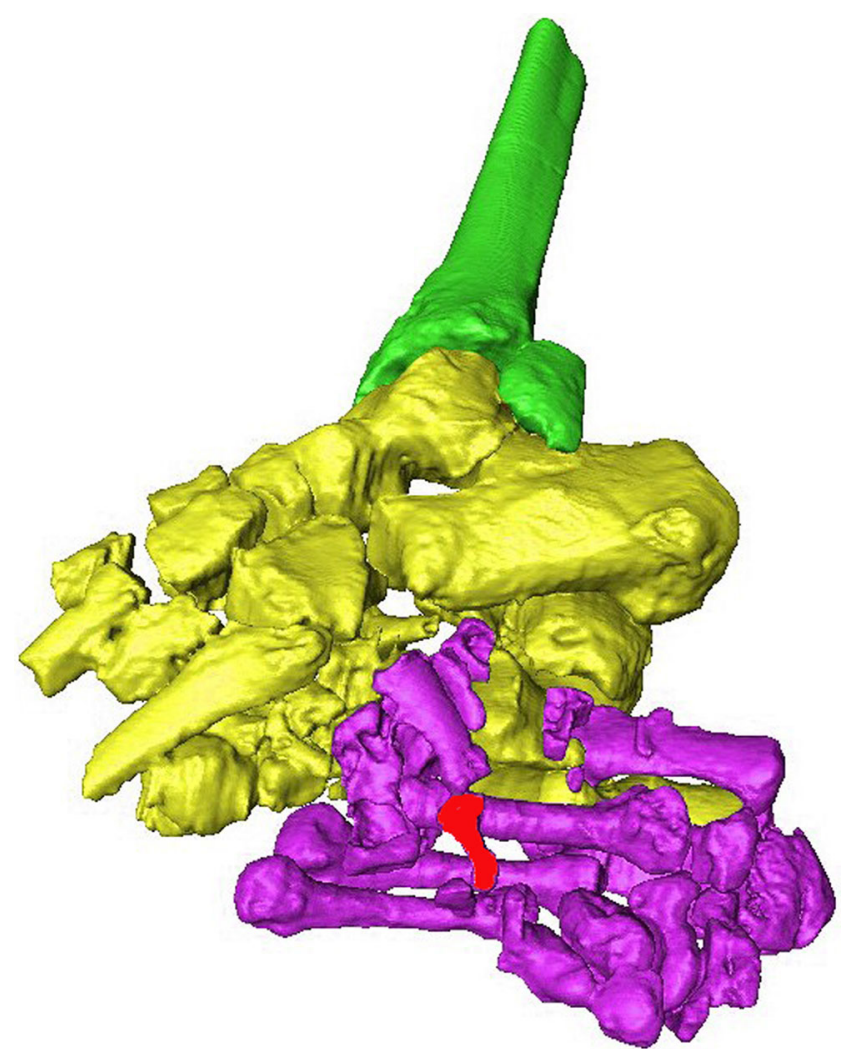

Fig. 4 Vue latérale gauche du bloc comprenant les extrémités distales de la fibula et du tibia gauches, les os des pieds et de la main droite après dégagement virtuel des ossements par imagerie 3D. Tibia et fibula (en vert), pieds (en jaune), main droite (en violet). La phalange distale de la main (rouge) est celle qui était totalement incluse dans le sédiment et qui a pu être identifiée grâce aux données CT. Capture d'écran sur Aviso 9.0.1 / Left lateral view of the block containing the distal ends of the left fibula, and tibia, bones from the feet and the right hand, virtually removed from the matrix by $3 \mathrm{D}$ imaging techniques. Tibia and fibula (green), foot (yellow), right hand (purple). The intermediate hand phalanx (red), embedded in the sediment, was discovered thanks to the CT-data. Generated by Aviso 9.0.1

mes thoracique et pelvien) témoignent d'un enfouissement précoce (antérieur à la décomposition des articulations) du cadavre dans le sédiment, alors qu'un séjour long dans l'eau conduit à une dislocation du squelette et à une dispersion des os en commençant par ceux des pieds et des mains [10]. Cependant, Duday $[8,11]$ décrit le corps d'une femme morte par noyade, dont le squelette est bien représenté (mains conservées jusqu'aux sésamoïdes) et les articulations, même labiles, sont maintenues en connexion stricte. Mais, dans le cas d'Asselar, la position du bas du corps (hanches fléchies et hyperflexion des genoux ramenant les pieds vers le bassin) est peu compatible avec l'hypothèse de la noyade, car les noyés (par accident) se présentent généralement en procubitus, membres étendus [10]. L'extrême flexion des genoux 
d'Asselar et la proximité pieds-bassin évoqueraient plutôt une position " donnée » au corps à l'occasion de sa mise en terre soit immédiatement après le décès, soit de 24 à 48 heures après, lorsque la rigidité cadavérique a disparu. Notons qu'une flexion prononcée des genoux semble commune aux sépultures de la région saharienne telles, en Tunisie, celles du site de SHM-1 à Hergla [12] et, au Mali, celles d'Hassi-el-Abiod [13] datées de 7000 BP.

Enfin, la proximité main droite-pieds observée à Asselar invite à s'interroger sur l'éventuelle présence d'une contention. En effet, ce cas a été récemment évoqué pour le site de Nataruk au Kenya (9500-10500 BP) où deux individus sont décrits, en raison du rapprochement étroit de leurs poignets et de la position de leurs mains, comme ayant eu les mains ligotées [14].

\section{Conclusion}

Cette étude, réalisée sur un individu issu de collections anciennes pour lequel nous disposions de nouvelles données grâce à la tomographie du bloc de sédiment comprenant les os des membres inférieurs (fibula gauche, tibias), des pieds et de la main droite, a permis de mieux définir les conditions de son enfouissement. Ainsi, la bonne représentation du squelette d'Asselar, la conservation de connexions articulaires, la position des membres inférieurs et des pieds et le maintien de la main droite au contact des pieds évoquent une attitude du corps qui semble incompatible avec l'hypothèse d'une noyade suivie d'un enfouissement accidentel, émise par Boule et Vallois [4]. Au contraire, nos observations, précisées par l'analyse des données tomographiques des os des extrémités des membres inférieurs encore pris dans le sédiment, permettent d'envisager un ensevelissement précoce du corps et la possibilité de son inhumation intentionnelle. En effet, la position rapprochée des deux pieds et la main droite aux doigts fléchis, placée sous le pied droit, impliquent une flexion prononcée des membres inférieurs donnée à l'individu peu de temps après son décès. La récurrence de cette position observée dans les sépultures d'un autre site malien de l'Holocène ancien, celui d'Hassiel-Abiod, sort le squelette d'Asselar de son isolement et incite à conclure à la pratique d'une sépulture.

Remerciements Les acquisitions ont été réalisées le 27 juillet 2013 à l'hôpital de la Pitié-Salpêtrière (Paris, 13) grâce à Mme Huynh-Charlier et à son équipe que nous remercions. Notre gratitude va également à Patricia Wils du plateau Accès scientifique à l'imagerie (ASIM) du Muséum national d'histoire naturelle qui a contribué au traitement des données tomographiques. Les résultats présentés sont en grande partie issus du Master (MNHN, QP) de Justine Dumesnil dont le stage a été financé par l'UMR 7194. Enfin, nous sommes très reconnaissants envers la SOGEDA pour son intérêt pour ce programme de recherche et le soutien procuré.

Liens d'intérêts : les auteurs déclarent ne pas avoir de lien d'intérêt.

\section{Références}

1. Augiéras EM, Draper WP, Gierzynski ME, et al (1931) D'Algérie au Sénégal. Mission Augiéras-Draper, 1927-1928. Société de géographie de Paris, $293 \mathrm{p}$

2. Petit-Maire D (2002) Sahara, sous le sable... des lacs. Un voyage dans le temps, CNRS Éditions, Paris, France, $127 \mathrm{p}$

3. Vialet A, André L, Aoudia L (2013) L'homme fossile d'Asselar (actuel Mali). Étude critique, mise en perspective historique et nouvelles interprétations. Anthropologie 117:345-61

4. Boule M, Vallois HV (1932) L'homme fossile d'Asselar (Sahara). Archives de l'Institut de paléontologie humaine, Paris, France, $90 \mathrm{p}$

5. Verneau R (1906) Les grottes de Grimaldi (Baoussé-Roussé). Anthropologie. Imprimerie de Monaco, tome 2, fascicule 1, $324 \mathrm{p}$

6. Legoux P (1962) Nouvelle étude anthropologique des « négroïdes de Grimaldi ». Comptes rendus des séances de l'Académie des sciences de Paris 255:2276-7

7. Duday H (1990) Observations ostéologiques et décomposition du cadavre : sépulture colmatée ou en espace vide. Rev Archeol Centre Fr, 29:193-6

8. Duday H (1995) Anthropologie de « terrain », archéologie de la mort. La mort, passé, présent, conditionnel, GVEP, La Rochesur-Yon, France, 33-58

9. Duday H, Courtaud P, Crubézy E, et al (1990) Anthropologie « de terrain » : reconnaissance et interprétation des gestes funéraires. Bull Mem Soc Anthropol Paris 2:29-49

10. Hamilton S J, Green M A (2017) Gross post-mortem changes in the human body. In: Schotsmans EMJ, Márquez-Grant N, Forbes SL (eds.) Taphonomy of human remains: forensic analysis of the dead and the depositional environment. John Wiley \& Sons, pp $11-25$

11. Duday H (2009) Archaeology of the dead. Oxbow Books, Oxford, Angleterre, $230 \mathrm{p}$

12. Munoz O, Candilio F, Roudesli-Chebbi S (2013) Les restes humains de SHM-1 (campagnes 2002-2007) : étude archéoanthropologique. In: Mulazzani S (Éd) Le capsien de Hergla (Tunisie). Culture, environnement, économie. Reports in African Archaeology. Africa Magna Verlag, Franckfurt, pp 299-314

13. Dutour O (1989) Hommes fossiles du Sahara : peuplements holocènes du Mali septentrional, Éditions du CNRS, Paris, France, $342 \mathrm{p}$

14. Mirazón Lahr M, Rivera F, Power R K, et al (2016) Inter-group violence among early Holocene hunter-gatherers of West Turkana, Kenya. Nature 529:394-8 